\title{
Novel cephalosporin conjugates display potent and selective inhibition of IMP type metallo- $\beta$-lactamases
}

\author{
Kamaleddin H. M. E. Tehrani, ${ }^{a}$ Nicola Wade, ${ }^{a}$ Vida Mashayekhi, ${ }^{b}$ Nora C. Brüchle, ${ }^{a}$ Willem \\ Jespers, ${ }^{c}$ Koen Voskuil, ${ }^{a}$ Diego Pesce, ${ }^{d, e}$ Matthijs van Haren, ${ }^{a}$ Gerard J. P. van Westen, ${ }^{c}$ \\ Nathaniel I. Martin ${ }^{a, *}$ \\ ${ }^{a}$ Biological Chemistry Group, Institute of Biology Leiden, Leiden University, Sylviusweg 72, 2333 BE Leiden, The \\ Netherlands.*n.i.martin@biology.leidenuniv.nl \\ ${ }^{b}$ Division of Cell Biology, Department of Biology, Faculty of Science, Utrecht University, Padualaan 8, 3584 CH \\ Utrecht, The Netherlands. \\ ${ }^{c}$ Division of Drug Discovery \& Safety, Leiden Academic Centre for Drug Research, Leiden University, Einsteinweg \\ 55, 2333 CC Leiden, The Netherlands. \\ ${ }^{d}$ Laboratory of Genetics, Wageningen University and Research, 6700 AA Wageningen, The Netherlands. \\ ${ }^{e}$ Department of Evolutionary Biology and Environmental Studies, University of Zurich, Winterthurerstrasse 190, \\ 8057 Zurich, Switzerland.
}




\begin{abstract}
In an attempt to exploit the hydrolytic mechanism by which $\beta$-lactamase enzymes degrade cephalosporins, we designed and synthesized a series of novel cephalosporin prodrugs aimed at delivering thiol-based inhibitors of metallo- $\beta$-lactamases (MBLs) in spatiotemporally controlled fashion. Notably, while enzyme-mediated hydrolysis of the $\beta$-lactam ring was found to occur, it was not accompanied by release of the thiol-based inhibitors. Nonetheless, the cephalosporin prodrugs, especially thiomandelic acid conjugate (8), demonstrated potent inhibition of IMP-type MBLs, with $\mathrm{IC}_{50}$ values in the nanomolar range. In addition, conjugate 8 was also found to greatly reduce the MIC of meropenem against an IMP-28 producing clinical isolate of K. pneumoniae. The results of kinetic experiments indicate that these prodrugs inhibit IMP-type MBLs by acting as slowly turned-over substrates. Structure-activity relationship studies revealed that both phenyl and carboxyl moieties of $\mathbf{8}$ are crucial for its potency. Furthermore, modeling studies indicate that productive interactions of the thiomandelic acid moiety of $\mathbf{8}$ with residues Trp28 and Lys161 within the IMP active site may contribute to the observed inhibitory potency and selectivity.
\end{abstract}




\section{Introduction}

Despite the growing threat of $\beta$-lactam resistance caused by metallo- $\beta$-lactamases (MBLs), there are no approved drugs on the market that target this class of enzymes. Unlike serine- $\beta$-lactamases, MBLs are metalloenzymes containing one or two zinc ions in their active site. An activated water molecule, coordinated by these zinc ions, in turn acts as the nucleophile in the hydrolysis all classes of $\beta$-lactams (except monobactams). ${ }^{1-3}$ MBLs of particular clinical significance are the New Delhi metallo- $\beta$-lactamase (NDM), Verona integron-encoded metallo$\beta$-lactamase (VIM) and imipenemase (IMP) families all of which possess broad $\beta$-lactamase activity. ${ }^{4}$ The previously reported inhibitors of MBLs have been the subject of several comprehensive review articles. ${ }^{5-8}$ Indeed, a wide range of compounds have been reported as MBL inhibitors with the majority acting by either sequestering zinc and/or by forming a ternary complex with metalloenzyme. ${ }^{9,10}$

Previously, we described the in vitro ability of a selected group of thiols (1-3, Figure 1A) to inhibit MBLs and in doing so resensitize a panel of MBL-producing clinical isolates to meropenem, a potent carbapenem antibiotic. ${ }^{11}$ Our earlier studies used isothermal titration calorimetry (ITC) to demonstrate that thiols 1 and 2 bind zinc with $K_{\mathrm{d}}$ values of $10 \mu \mathrm{M}$ and 20 $\mu \mathrm{M}$ respectively. However, as we also demonstrated, these thiol-containing compounds are prone to rapid oxidation to their corresponding disulfides, leading to the loss of zinc-binding affinity, MBL inhibition, and synergistic activity. ${ }^{11}$ Recently, we reported a cephalosporin prodrug approach to selectively enable the release of strong zinc-chelating small molecules upon hydrolysis by MBLs. ${ }^{12}$ In doing so, we identified inhibitors with potent activity against NDMand VIM-type MBLs. In the present study, we aimed to apply a similar design strategy employing thiol-based MBL inhibitors 1-3. As illustrated in Figure 1B, the prodrugs consist of a cephalosporin core with the thiol-based MBL inhibitors linked at the 3-position. The hydrolytic action of MBLs upon such conjugates was envisioned to result in a cascade reaction ultimately leading to release of the inhibitor with both special and temporal control. We hypothesized that in the case of thiol-based inhibitors 1-3, this prodrug strategy could be effective by addressing not only the selectivity but also their poor stability stemming from their rapid oxidation. Here we describe the preparation of cephalosporin-thiol conjugates 6, 8-10 and evaluation of their performance as MBL-inhibitor prodrugs capable of resensitizing MBL-expressing strains to $\beta$-lactam antibiotics. 
A)

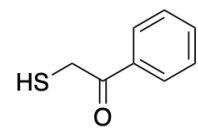

1

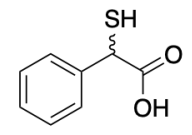

2

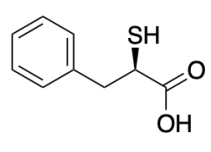

$3 a$

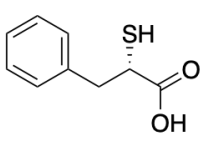

3b

B)
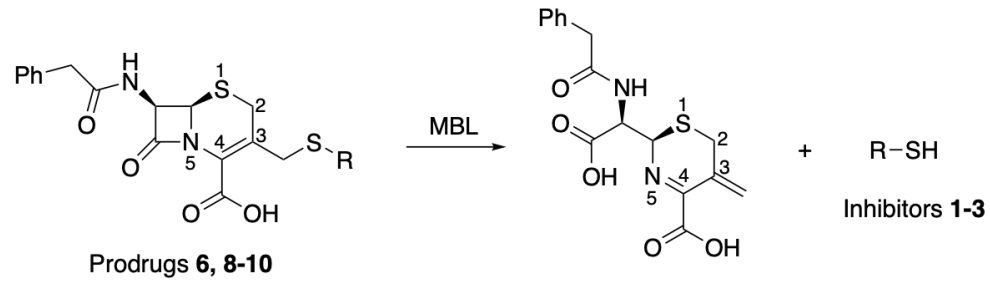

9, R: $\overbrace{\mathrm{Ph}}^{\mathrm{O}}$ 10, R: $\underbrace{\mathrm{O}}_{\mathrm{Ph}}$

Figure 1. A) The previously reported thiols as MBL-inhibitors. B) Cephalosporin prodrugs of the thiols 1-3 and their related structural analogs.

\section{Results and discussion}

The cephalosporin-thiol conjugates were synthesized using two different routes (Scheme 1). Thioalkylation of mercaptoacetophenone with the chloromethyl cephalosporin "GCLE" (4), a common intermediate used in the industrial synthesis of cephalosporin antibiotics, yielded intermediate $\mathbf{5}$ followed by deprotection with TFA to yield compound $\mathbf{6}$. Alternatively, compounds 8-13 were prepared via the $\mathrm{BF}_{3}$-promoted substitution of 7-aminocephalosporanic acid (7-ACA, 7) with the corresponding thiols, followed by acylation of the 7-amino group (see the experimental section for detailed procedures). Notably, conjugate $\mathbf{8}$ was prepared as a diastereomeric mixture given the stereochemical instability of the corresponding thiomandelic acid building block $2 .{ }^{13}$ Compounds 11-13 as well as $\mathbf{1 5}$ were designed and synthesized for the purpose of structure-activity relationship evaluation of the thiol conjugates 6 and 8-10. 

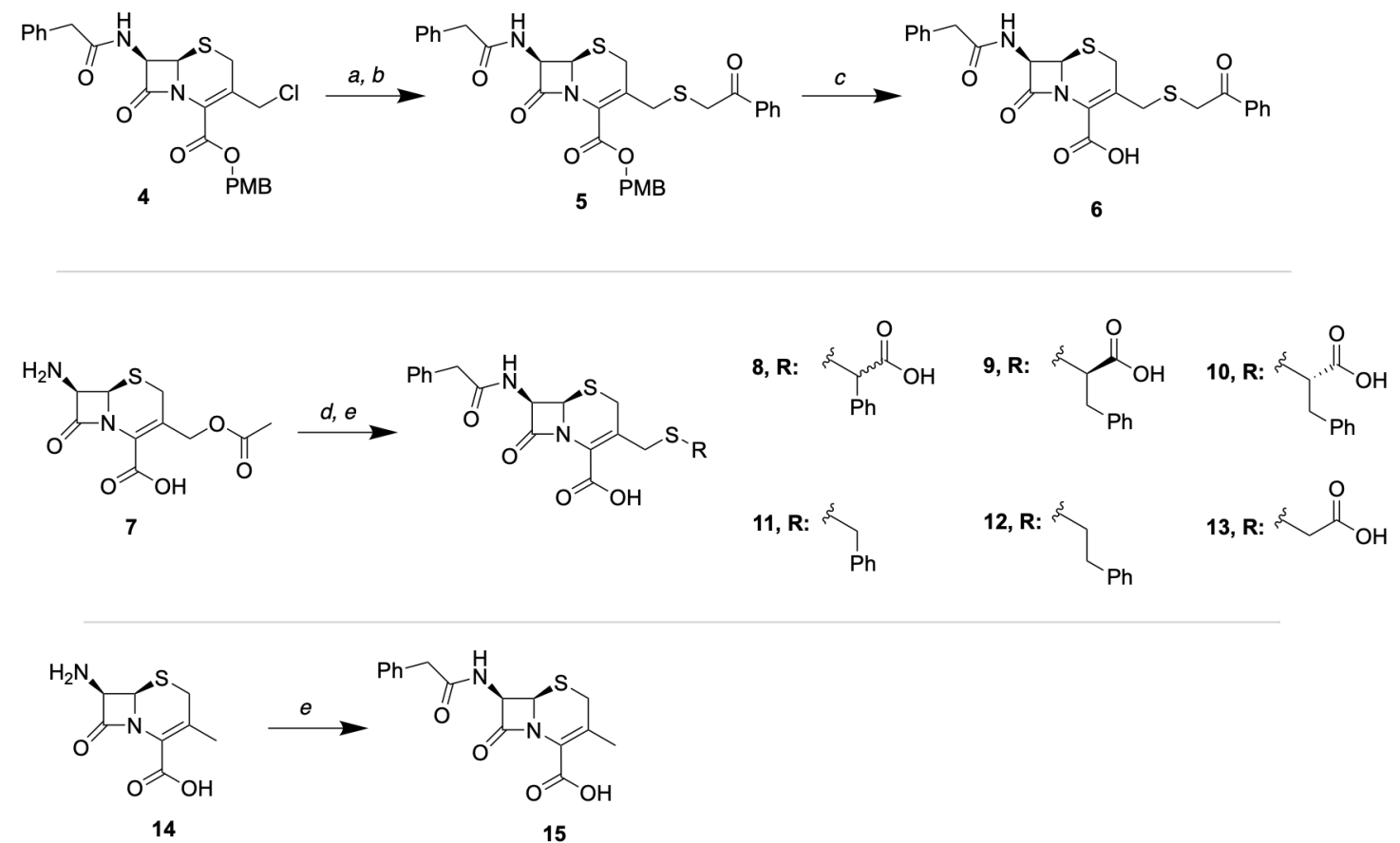

Scheme 1. Chemical route to the cephalosporin conjugates. Reagents and conditions: a. NaI, DMF, r.t., 30 min.; b. $\mathrm{NaHCO}_{3}, 1$, r.t., $20 \mathrm{~h}$; c. TFA, anisole, $0{ }^{\circ} \mathrm{C}, 1 \mathrm{~h}$; d. $\mathrm{BF}_{3} \cdot \mathrm{OEt}_{2}$, thiols, $\mathrm{ACN}, 45{ }^{\circ} \mathrm{C}, 2 \mathrm{~h}$; e. phenylacetyl chloride, saturated $\mathrm{NaHCO}_{3}$ solution, acetone, r.t., $20 \mathrm{~h}$.

To assess the zinc-binding properties of the MBL-inhibitor prodrugs, ITC binding studies were performed which revealed no appreciable binding interaction with zinc. This was in contrast with the starting thiols which were found to be relatively strong zinc-binders with low- $\mu \mathrm{M} K_{\mathrm{d}}$ values $^{11}$. In addition, stability analyses were performed to test whether inhibitor release occurred spontaneously. Following overnight incubation in Mueller-Hinton broth, HPLC analysis of the conjugates $\mathbf{6}$ and $\mathbf{8 - 1 0}$ showed that all demonstrated good stability ( $>95 \%$ intact after $15 \mathrm{~h}$, Table S1).

The compounds were next tested for their ability to restore the antibacterial activity of meropenem against a panel of MBL-producing clinical isolates. The results showed that compound 8 and 9 were potent synergists, lowering the MIC of meropenem against IMP-producing isolates most effectively (Table 1). 
Table 1. MIC of meropenem in combination with the cephalosporin conjugates tested at multiple concentrations against 4 clinical isolates.

\begin{tabular}{|c|c|c|c|c|c|}
\hline \multirow[b]{2}{*}{ Compound } & \multirow[b]{2}{*}{$\begin{array}{l}\text { Concentration } \\
(\mu \mathrm{g} / \mathrm{mL})\end{array}$} & \multicolumn{4}{|c|}{ Meropenem MIC $(\mu \mathrm{g} / \mathrm{mL})$} \\
\hline & & $\begin{array}{l}\text { E. coli } \\
\text { (IMP-4) }\end{array}$ & $\begin{array}{l}\text { K. pneumoniae } \\
\text { (IMP-28) }\end{array}$ & $\begin{array}{l}\text { E. coli } \\
\text { (VIM-2) }\end{array}$ & $\begin{array}{l}\text { E. coli } \\
\text { (NDM-1) }\end{array}$ \\
\hline \multirow[t]{4}{*}{6} & 0 & 2 & 4 & 8 & 32 \\
\hline & 32 & $0.25(8)^{a}$ & $1(4)$ & $8(1)$ & $16(2)$ \\
\hline & 64 & $0.125(16)$ & $0.5(8)$ & $4(2)$ & $8(4)$ \\
\hline & 128 & $0.125(16)$ & $0.25(16)$ & $4(2)$ & $8(4)$ \\
\hline \multirow[t]{4}{*}{8} & 0 & 2 & 4 & 8 & 32 \\
\hline & 32 & $\leq 0.063(\geq 32)$ & $\leq 0.063(\geq 64)$ & $4(2)$ & $16(2)$ \\
\hline & 64 & $\leq 0.063(\geq 32)$ & $\leq 0.063(\geq 64)$ & $2(4)$ & $16(2)$ \\
\hline & 128 & $\leq 0.063(\geq 32)$ & $\leq 0.063(\geq 64)$ & $1(8)$ & $8(4)$ \\
\hline \multirow[t]{4}{*}{9} & 0 & 2 & 4 & 8 & 32 \\
\hline & 32 & $\leq 0.063(\geq 32)$ & $\leq 0.063(\geq 64)$ & $4(2)$ & $16(2)$ \\
\hline & 64 & $\leq 0.063(\geq 32)$ & $\leq 0.063(\geq 64)$ & $4(2)$ & $8(4)$ \\
\hline & 128 & $\leq 0.063(\geq 32)$ & $\leq 0.063(\geq 64)$ & $2(4)$ & $8(4)$ \\
\hline \multirow[t]{4}{*}{10} & 0 & 4 & 4 & 8 & 64 \\
\hline & 32 & $0.25(16)$ & $1(4)$ & $8(1)$ & $32(2)$ \\
\hline & 64 & $0.125(32)$ & $0.5(8)$ & $8(1)$ & $32(2)$ \\
\hline & 128 & $0.125(32)$ & $0.25(16)$ & $4(2)$ & $16(4)$ \\
\hline \multirow[t]{4}{*}{11} & 0 & 4 & 2 & 4 & 32 \\
\hline & 32 & $0.25(16)$ & $0.5(4)$ & $2(2)$ & $16(2)$ \\
\hline & 64 & $0.125(32)$ & $0.25(8)$ & $2(2)$ & $16(2)$ \\
\hline & 128 & $0.125(32)$ & $0.25(8)$ & $1(4)$ & $16(2)$ \\
\hline \multirow[t]{4}{*}{12} & 0 & 4 & 4 & 8 & 64 \\
\hline & 32 & $0.25(16)$ & $0.5(8)$ & $8(1)$ & $32(2)$ \\
\hline & 64 & $0.25(16)$ & $0.25(16)$ & $4(2)$ & $16(4)$ \\
\hline & 128 & $0.125(32)$ & $0.25(16)$ & $4(2)$ & $16(4)$ \\
\hline \multirow[t]{4}{*}{13} & 0 & 4 & 2 & 4 & 32 \\
\hline & 32 & $\leq 0.063(\geq 64)$ & $0.25(8)$ & $2(2)$ & $16(2)$ \\
\hline & 64 & $\leq 0.063(\geq 64)$ & $0.25(8)$ & $2(2)$ & $16(2)$ \\
\hline & 128 & $\leq 0.063(\geq 64)$ & $0.125(16)$ & $1(4)$ & $8(4)$ \\
\hline \multirow[t]{4}{*}{15} & 0 & 2 & 2 & 4 & 32 \\
\hline & 32 & $0.5(4)$ & $1(2)$ & $2(2)$ & $16(2)$ \\
\hline & 64 & $0.25(8)$ & $0.5(4)$ & $2(2)$ & $16(2)$ \\
\hline & 128 & $0.25(8)$ & $0.25(8)$ & $1(4)$ & $16(2)$ \\
\hline \multirow[t]{4}{*}{ DPA } & 0 & 4 & 2 & 4 & 32 \\
\hline & 32 & $\leq 0.063(\geq 64)$ & $0.125(16)$ & $\leq 0.063(\geq 64)$ & $0.5(64)$ \\
\hline & 64 & $\leq 0.063(\geq 64)$ & $\leq 0.031(\geq 64)$ & $\leq 0.063(\geq 64)$ & $0.5(64)$ \\
\hline & 128 & $\leq 0.063(\geq 64)$ & $\leq 0.031(\geq 64)$ & $\leq 0.063(\geq 64)$ & $\leq 0.5(\geq 64)$ \\
\hline
\end{tabular}

${ }^{a}$ Fold reduction of MIC shown in brackets 
Encouraged by the promising results against the MBL-producing clinical isolates, we tested the ability of the conjugates to inhibit purified IMP-1, IMP-28, VIM-2, and NDM-1 enzymes. The biochemical assay used for these studies employed the chromogenic cephalosporin nitrocefin as substrate. ${ }^{8}$ The $\mathrm{IC}_{50}$ data obtained (Table 2) are consistent with the trends observed in the bacterial growth inhibition synergy assays, with strains possessing IMP-type enzymes being most strongly inhibited by the conjugates with $\mathbf{8}$ and $\mathbf{9}$.

Table 2. $\mathrm{IC}_{50}(\mu \mathrm{M})$ of cephalosporin conjugates reported as mean $\pm \mathrm{SD}^{a}$

\begin{tabular}{lllll}
\hline Compound & IMP-1 & IMP-28 & NDM-1 & VIM-2 \\
\hline $\mathbf{6}$ & $3.3 \pm 0.2$ & $14 \pm 1$ & $77 \pm 12$ & $76 \pm 11$ \\
$\mathbf{8}$ & $0.47 \pm 0.08$ & $0.46 \pm 0.04$ & $>100$ & $10 \pm 0.5$ \\
$\mathbf{9}$ & $4.7 \pm 0.4$ & $1.1 \pm 0.2$ & $94 \pm 0.2$ & $16 \pm 1$ \\
$\mathbf{1 0}$ & $48 \pm 0.7$ & $30 \pm 9$ & $51 \pm 1$ & $19 \pm 1$ \\
$\mathbf{1 1}$ & $43 \pm 3$ & $45 \pm 5$ & $>100$ & $73 \pm 0.1$ \\
$\mathbf{1 2}$ & $2.2 \pm 0.4$ & $4.3 \pm 0.8$ & $>100$ & $49 \pm 3$ \\
$\mathbf{1 3}$ & $>100$ & $>100$ & $72 \pm 2$ & $53 \pm 12$ \\
$\mathbf{1 5}$ & $>100$ & $>100$ & $>100$ & $57 \pm 9$ \\
DPA & $29 \pm 0.5$ & $29 \pm 5$ & $10 \pm 0.1$ & $10 \pm 0.8$ \\
\hline
\end{tabular}

${ }^{a}$ Nitrocefin was used as the chromogenic substrate. A detailed description of the assay can be found in the experimental section.

To investigate the mechanism of inhibition, and more specifically to assess release of the thiol inhibitors, the most potent cephalosporin conjugates 8 and $\mathbf{9}$ were incubated with IMP-28 and analyzed using ${ }^{1} \mathrm{H}-\mathrm{NMR}$ and LC-MS techniques. It has been shown previously by our group and others that the molecular mechanism of cephalosporin hydrolysis can be probed in situ using NMR techniques. ${ }^{12,14,15}$ In the present study, we used the commercially available 7-phenylacetylamide derivative of 7-ACA (compound 16, Figure 2A) as a positive control. After incubating this compound with IMP-28, the rapid appearance of vinylic protons corresponding to the elimination product were detected at $c a .5 .50 \mathrm{ppm}$ (Figure 2A). However, when $\mathbf{8}$ and $\mathbf{9}$ were subjected to the same experiment, these vinylic signals were not detected (Figure 2B) suggesting that the thiols at the 3-position were not being released. The results of these ${ }^{1} \mathrm{H}-\mathrm{NMR}$ studies were further corroborated by LC-MS analyses of the hydrolysis products which revealed the 
hydrolyzed $\beta$-lactam compounds $\mathbf{8 H}$ and $\mathbf{9 H}$ as the only detectable products (Figure $2 \mathrm{C}$, see supporting information Figures S2-S4 and S6 for complete NMR and LC-MS data).
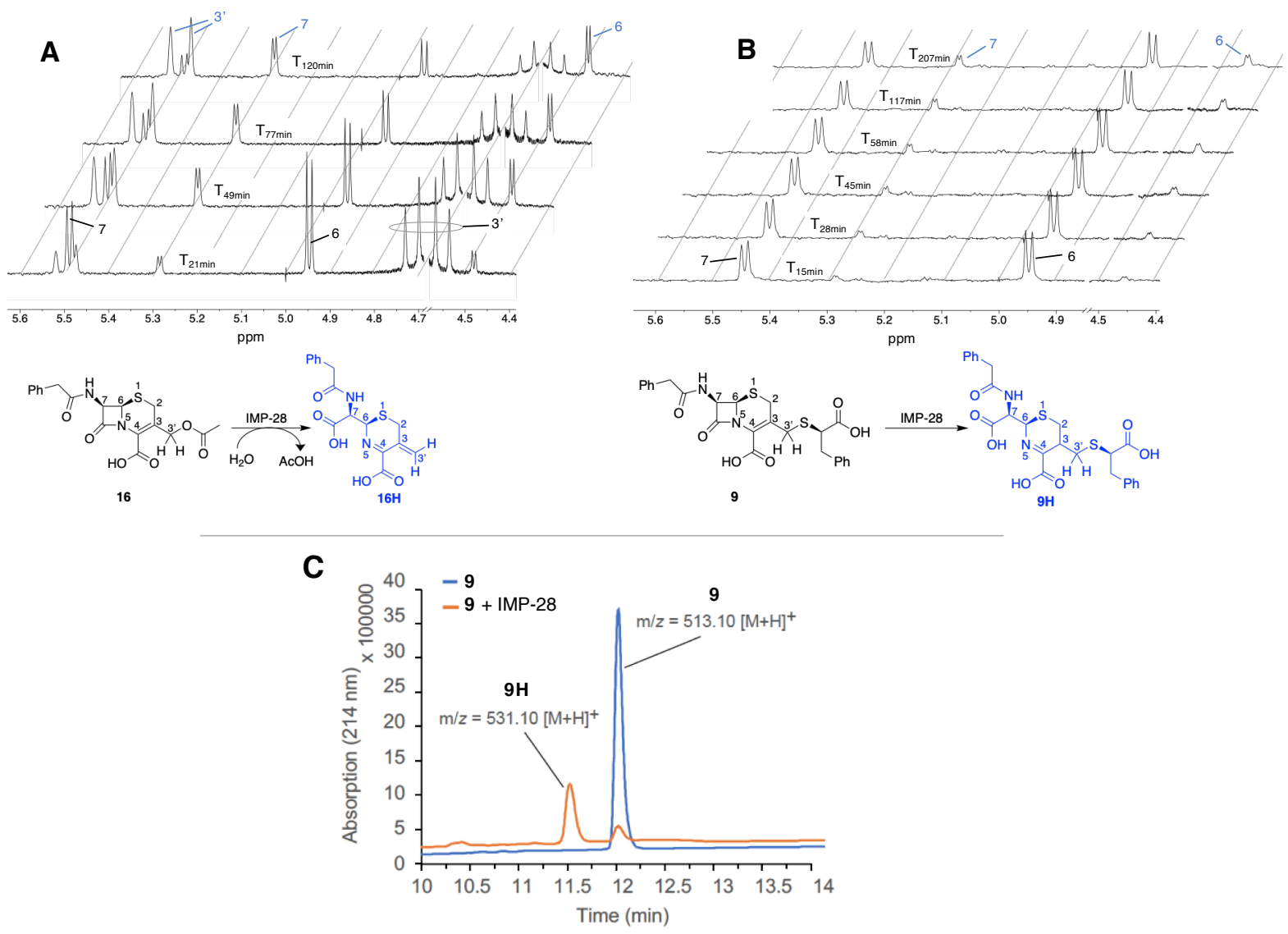

Figure 2. A) Enzymatic degradation of $\mathbf{1 6}$ showing the growth of the signals corresponding to the vinylic protons of $\mathbf{1 6 H}$ resonating as 2 singlets $c a .5 .5 \mathrm{ppm}$. B) Enzymatic degradation of 9 instead leads to $\mathbf{9 H}$. For the purpose of clarity, the segment corresponding to water signal has been omitted from the NMR spectra. C) LC-MS analysis of IMP-28-mediated degradation of $\mathbf{9}$, confirms the exclusive formation of $\mathbf{9 H}$. 
The finding that compounds $\mathbf{8}$ and $\mathbf{9}$ demonstrate potent inhibition of IMP-28 despite not releasing the corresponding zinc-binding thiol inhibitors upon MBL-mediated $\beta$-lactam hydrolysis was surprising. To better understand the mechanism of inhibition of these cephalosporin conjugates we next determined the kinetic parameters of the hydrolysis of the cephalosporin conjugates using purified MBL enzymes. In addition, we evaluated the MBLinhibitory activity of the partial hydrolysis products $\mathbf{8 H}$ and $\mathbf{9 H}$. The kinetic analysis of the hydrolysis of the cephalosporins by IMP-28, NDM-1 and VIM-2 provided valuable insights on the observed IMP-28 selectivity for the inhibitors and the greater potency of $\mathbf{8}$ and $\mathbf{9}$. These analyses showed that IMP-28 has the lowest catalytic efficiency for 8 and $\mathbf{9}$ among the tested cephalosporins (see Table 3 for relative $k_{\text {cat }} / K_{\mathrm{M}}$ data). Comparison with the other major MBL families also revealed that $\mathbf{8}$ and $\mathbf{9}$ were hydrolyzed more efficiently by NDM-1 and VIM-2 than by IMP-28. These findings indicate that conjugates 8 and $\mathbf{9}$ inhibit IMP-28 either by acting as slowly turned-over substrates and/or that the hydrolyzed products $\mathbf{8 H}$ and $\mathbf{9 H}$ are more tightly bound within the IMP active site than either the NDM or VIM active sites.

Table 3. The Michaelis-Menten parameters determined for the cephalosporin conjugates as substrates of IMP-28, VIM-2 and NDM-1. ${ }^{a, b}$

\begin{tabular}{llllll}
\hline Enzyme & Substrate & $K_{\mathrm{M}}(\mu \mathrm{M})$ & $k_{\text {cat }}\left(\mathrm{s}^{-1}\right)$ & $k_{\text {cat }} / K_{\mathrm{M}}\left(\mu \mathrm{M}^{-1} \cdot \mathrm{s}^{-1}\right)$ & $\begin{array}{l}\text { Relative } \\
k_{\text {cat }} / K_{\mathrm{M}}\end{array}$ \\
\hline NDM-1 & $\mathbf{8}$ & $14.0 \pm 2$ & 13.1 & 0.936 & 100 \\
& $\mathbf{9}$ & $21.2 \pm 4$ & 17.4 & 0.821 & 88 \\
\hline VIM-2 & $\mathbf{8}$ & $8.28 \pm 2$ & 4.06 & 0.490 & 52 \\
& $\mathbf{9}$ & $4.30 \pm 1$ & 2.35 & 0.546 & 58 \\
\hline IMP-28 & $\mathbf{8}$ & $129 \pm 13$ & 0.386 & 0.003 & 0.30 \\
& $\mathbf{9}$ & $249 \pm 7$ & 2.83 & 0.011 & 1.2 \\
& $\mathbf{1 0}$ & $175 \pm 19$ & 41.0 & 0.234 & 44 \\
& $\mathbf{1 1}$ & $20.5 \pm 4$ & 10.8 & 0.529 & 56 \\
& $\mathbf{1 2}$ & $83.3 \pm 16$ & 6.12 & 0.073 & 14 \\
& $\mathbf{1 3}$ & $219 \pm 23$ & 37.0 & 0.169 & 18 \\
& $\mathbf{1 5}$ & $392 \pm 73$ & 23.3 & 0.059 & 6.3 \\
\hline
\end{tabular}

${ }^{a}$ The experimental procedure has been described in detail in the experimental section.

${ }^{b}$ See supporting information for the Michaelis-Menten graphs. 
To evaluate the inhibitory activity of the hydrolysis products $\mathbf{8 H}$ and $\mathbf{9 H}$, the intact conjugates 8 and 9 were first fully hydrolyzed by incubation with NDM-1 as described in the experimental section (see Figure S7 for the LC-MS traces). Following hydrolysis, the NDM-1 enzyme was completely removed via spin-filtration as confirmed by the lack of nitrocefin activity by the filtrate. The partially hydrolyzed $\mathbf{8 H}$ and $\mathbf{9 H}$ were then tested for their inhibition of MBLs. Interestingly, both hydrolysis products were found to possess potent activity against IMP-1 and IMP-28 with sub- $\mu$ M IC 50 values (Table S2). In addition, the hydrolysis products $\mathbf{8 H}$ and 9H were evaluated for their zinc-binding affinity using ITC. When zinc was titrated into the solution of 8 and 9 preincubated with NDM-1, a binding interaction with $K_{\mathrm{d}}$ values of $9.67 \mu \mathrm{M}$ and $3.19 \mu \mathrm{M}$ were observed respectively (Figure 6), while the intact cephalosporins showed no zinc-binding affinity. This affinity for zinc binding may therefore also contribute to the inhibitory activity of $\mathbf{8 H}$ and $\mathbf{9 H}$.
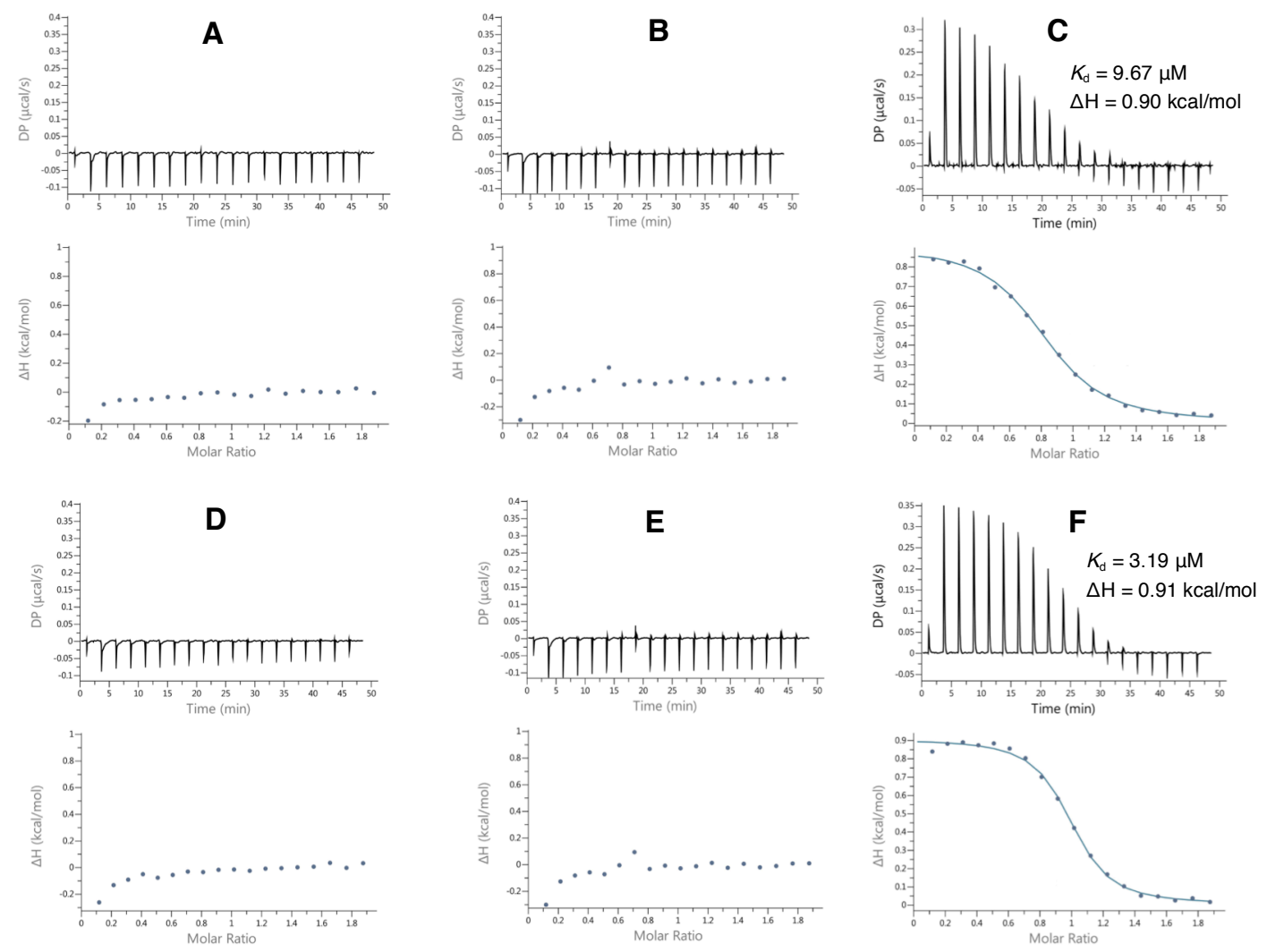

Figure 6. ITC thermograms resulting from titration of $\mathrm{ZnSO}_{4}$ into a solution containing: A) Compound 8; B) purified NDM-1; C) Compound 8H; D) Compound 9; E) purified NDM-1; F) Compound $\mathbf{9 H}$. 
The $\mathrm{IC}_{50}$ data obtained for the various conjugates prepared also provides some structureactivity insights (Table 2). Specifically, elimination of the carboxylic acid (11), phenyl group (13), or the entire thiomandelic acid fragment (15), causes the activity against IMP-1 and IMP-28 to be decreased at least by $\sim 100$ times, suggesting that the thiomandelic acid fragment introduces productive binding interactions with the IMP active site. Also interesting was the observation that compound 9 was $\sim 10$-fold and $\sim 30$-fold more potent than its diastereomer 10 against IMP-1 and IMP-28 respectively.

In an attempt to provide further insights into the binding mode of the most potent compound (8), a computational model was derived based on docking of this compound to the published crystal structure of IMP-1. ${ }^{16}$ The docking hypothesis is based on the high resemblance of $\mathbf{8 H}$ to the hydrolysis product of cephalexin, a compound previously co-crystallized with NDM-1. ${ }^{15}$ Thus, we overlaid the IMP-1 and NDM-1 structures, and used the maximum common substructure (MCS, see methods) between $\mathbf{8 H}$ and the hydrolysis product of cephalexin. The resulting docking pose aligned well with $\mathbf{8 H}$, as well as other representative hydrolysis products presented in this work. Noteworthy is the fact that both diastereomers of $\mathbf{8 H}$, which could not be separated for the assay, are accommodated in the binding site and in similar fashion (see Figure S8). We next studied the docking of the parent compound $\mathbf{8}$ which revealed some interesting findings in comparison with its hydrolysis product $\mathbf{8 H}$ : In compound $\mathbf{8}$ (Figure 7A), the zinc ions are anchored by the carboxylate on cephem C-4 together with the carboxylate from the thiomandelic acid moiety. The latter in $\mathbf{8 H}$ (figure 7B) is replaced by the carboxylate resulted from $\beta$-lactam hydrolysis. Also notable for $\mathbf{8 H}$ is the way the phenyl group of thiomandelic acid unit interacts with Trp28 and Leu165 residues. The binding mode of $\mathbf{8}$ suggests that this phenyl group can engage in a $\pi-\pi$ interaction with Trp28. Upon hydrolysis to $\mathbf{8 H}$, however, this phenyl group is predicted to move away from Trp28 and towards Leu165 where it can form hydrophobic interactions in a pocket around that residue. Another interesting potential binding interaction detected in the modeling studies involves Lys161. This residue can from a salt bridge with the thiomandelic acid carboxylate in both $\mathbf{8}$ and $\mathbf{8 H}(2.4 \AA$ distance). This may explain the $\sim 100$-fold loss of activity against IMP enzymes when the carboxylate of $\mathbf{8}$ is eliminated (as in 11). The Lys161 interaction can also contribute to the IMP-selectivity of compound $\mathbf{8}$, since in NDM-1 this Lys is predicted to be $3.6 \AA$ from the thiomandelic acid carboxylate. A second factor that may contribute to IMP selectivity for $\mathbf{8}$ is an interaction with Trp28. An analysis of all 
available IMP-1 structures (Figure 7C) indicate that the Trp29 side chain is part of a flexible loop region that can form a cage around the binding site, optimally accommodating compound $\mathbf{8}$. Interestingly, this residue is replaced by Phe70 in NDM-1 where it is part of a more flexible region as observed in the analysis of all X-ray structures for NDM-1 (figure 7D). These differences may contribute to a weaker binding for compound 8 in the NDM-1 active site providing further understanding of the IMP-specific inhibition observed.
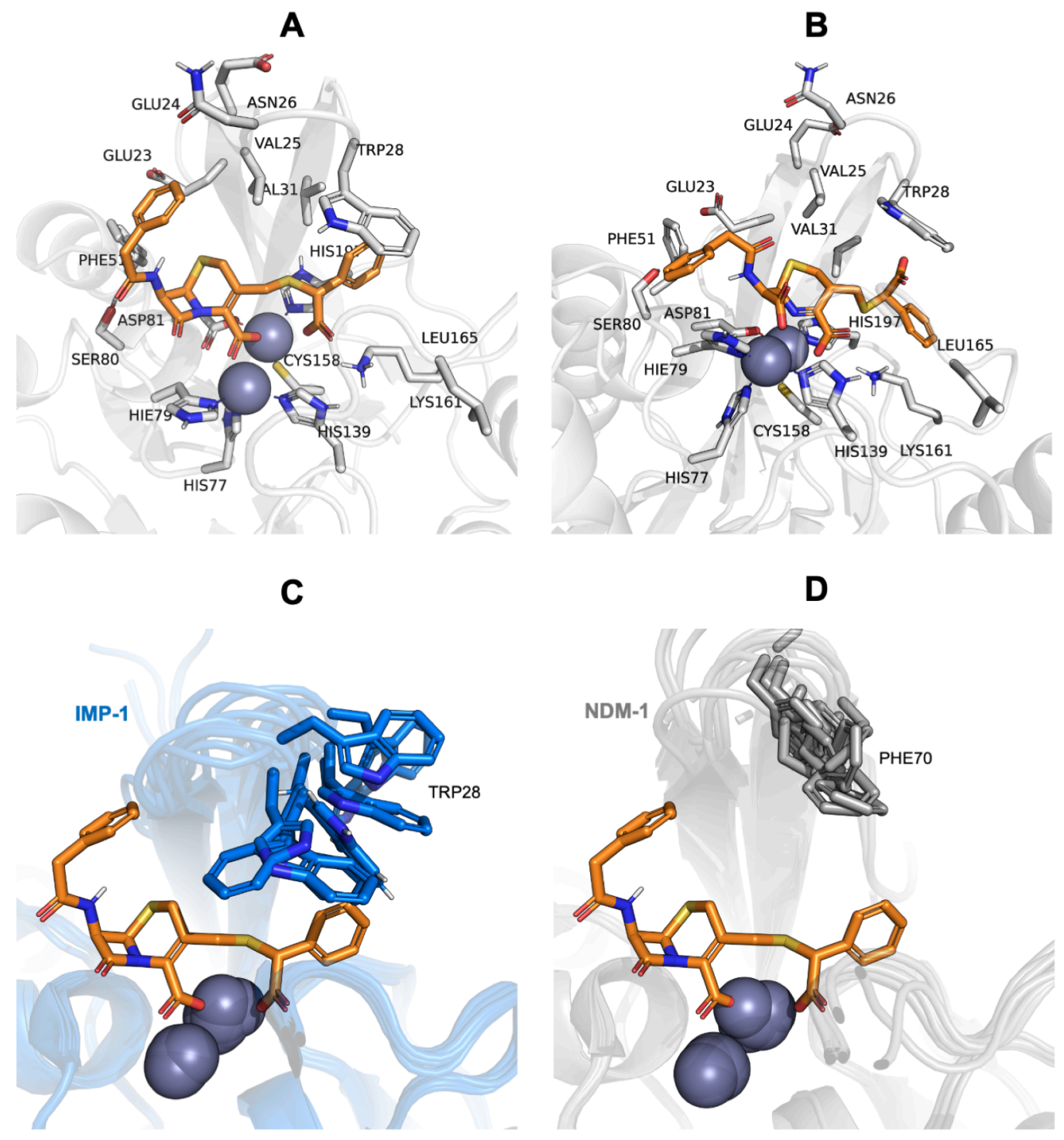

Figure 7. Modeling studies of compound $\mathbf{8}$ docked into the active site of IMP-1 and NDM-1. For simplicity only the diastereomer of $\mathbf{8}$ containing $R$-thiomandelic acid moiety has been shown. A) Compound $\mathbf{8}$ in the active site of IMP-1; B) Compound $\mathbf{8 H}$ in the active site of IMP-1; C) The docking model of compound $\mathbf{8}$ shown in an ensemble of overlaid X-ray structures of IMP-1; D) The docking model of compound $\mathbf{8}$ shown in an ensemble of overlaid X-ray structures of NDM-1. 


\section{Conclusion}

We here describe a series of cephalosporin-based MBL inhibitor prodrugs designed to release zinc-chelating small molecule thiols upon MBL-mediated hydrolysis. Notably, while displaying potent inhibition of IMP-type MBLs, these conjugates did not function as mechanistically predicted. While MBL-mediated hydrolysis was observed, the release of the small molecule thiol fragments did not spontaneously occur for the conjugates included in this study. This lack of release is presumably due to the $\mathrm{p} K_{\mathrm{a}}$ of the corresponding thiols not being low-enough to enable them to behave as leaving groups. Nonetheless, the finding that the cephalosporin conjugates (6, 8 and 9) selectively inhibit IMP enzymes is notable. Based on kinetic analyses, the most potent conjugates $\mathbf{8}$ and $\mathbf{9}$ were shown to be slowly turned-over substrates of IMP-28. In addition, the hydrolysis products $\mathbf{8 H}$ and $\mathbf{9 H}$ were found to be IMPselective inhibitors. Our findings suggest that the IMP inhibition observed with compounds 8 and 9 may be due to a combination of effects whereby the slowly turned-over substrate and the resulting hydrolysis product both contribute to enzyme inhibition. Furthermore, modeling studies indicate that the interaction of $\mathbf{8} / \mathbf{8 H}$ and $\mathbf{9 / 9 H}$ with the IMP active site residues Trp28, Leu165, and Lys 161 contribute to the observed potency and IMP-selectivity. These finding can guide the future optimization efforts to further improve the potency as well as broaden the spectrum of MBL inhibition exerted by the next-generation cephalosporin-based MBL inhibitors. 


\section{Experimental section}

General. Compound 4 (GCLE), 7-ACA (7) and 7-ADCA (14) were purchased from Combi-Blocks (US) and nitrocefin from Cayman chemical. The preparation of thiols 1-3 was performed as previously described. ${ }^{11}$ Compound 16 was synthesized via the acylation of 7-ACA following a previously reported procedure. ${ }^{17}$ Proton and carbon nuclear magnetic resonance spectra were recorded on an AV400 NMR spectrometer (Bruker) and samples were dissolved in $\mathrm{CDCl}_{3}$ or DMSO- $d_{6}$. HRMS analyses were performed on a Thermo Scientific Dionex UltiMate 3000 HPLC system with a Phenomenex Kinetex C18 column $(2.1 \times 150 \mathrm{~mm}, 2.6 \mu \mathrm{m})$ at $35^{\circ} \mathrm{C}$ and equipped with a diode array detector. The samples were eluted over a gradient of solution A $(0.1 \%$ formic acid in water) vs. solution B $(0.1 \%$ formic acid in acetonitrile). This system was connected to a Bruker micrOTOF-Q II mass spectrometer (ESI ionization) calibrated internally with sodium formate.

Compound 6. GCLE (4, $1.0 \mathrm{~g}, 2.1 \mathrm{mmol})$ and $\mathrm{NaI}(314 \mathrm{mg}, 2.1 \mathrm{mmol})$ were stirred in DMF $(10 \mathrm{~mL})$ for $30 \mathrm{~min}$ at room temperature. Then mercaptoacetophenone (479 $\mathrm{mg}, 3.15$ mmol) and sodium bicarbonate $(200 \mathrm{mg}, 2.38 \mathrm{mmol})$ were added successively, and the mixture was stirred overnight. The reaction mixture was then partitioned between water and DCM followed by washing the organic layer with brine $(3 \times 20 \mathrm{~mL})$. Concentration of the organic layer and purification of the residue on silica using ethyl acetate and DCM mixture as eluent furnished 5 as a pale yellow solid (854 mg, 68\%). ${ }^{1} \mathrm{H}$ NMR (400 $\left.\mathrm{MHz}, \mathrm{CDCl}_{3}\right): \delta 7.89(\mathrm{~d}, J=8.3 \mathrm{~Hz}$, aromatic $\mathrm{H}, 1 \mathrm{H}), 7.58(\mathrm{t}, J=8.0 \mathrm{~Hz}$, aromatic $\mathrm{H}, 1 \mathrm{H}), 7.45(\mathrm{t}, J=8.0 \mathrm{~Hz}$, aromatic H, 2H), 7.37$7.25(\mathrm{~m}$, aromatic $\mathrm{H}, 7 \mathrm{H}), 6.85(\mathrm{dd}, J=8.6 \mathrm{~Hz}, J=1.8 \mathrm{~Hz}$, aromatic $\mathrm{H}, 2 \mathrm{H}), 5.99(\mathrm{~d}, J=9.2 \mathrm{~Hz}$, 1H), 5.77 (m, $\beta$-lactam C-H, 1H), 5.14 (s, benzyloxy $\left.\mathrm{CH}_{2}, 2 \mathrm{H}\right), 4.90$ (d, J=4.9 Hz, 1H), 3.993.45 (m, aliphatic H, $11 \mathrm{H}),{ }^{13} \mathrm{C}$ NMR (101 MHz, DMSO- $\left.d_{6}\right): \delta 194.41,171.14,164.50,161.52$, 159.83 , 135.37, 133.72, 133.46, 130.67, 129.40, 129.10, 128.69, 128.53, 128.49, 127.64, 126.78, $124.58,113.91,67.93,59.03,57.74,55.23,43.26,37.81,33.81,27.72$. HRMS (ESI): $[\mathrm{M}+\mathrm{H}]^{+}$ calculated: 603.1624, found: 603.1620. To 5 (600 mg, $1.0 \mathrm{mmol})$ was added TFA/anisole (15 $\mathrm{mL} / 3 \mathrm{~mL}$ ) and the mixture was stirred at $0{ }^{\circ} \mathrm{C}$ for $1 \mathrm{~h}$. It was then concentration under vacuum and the residue was precipitated by 1:1 mixture of diethyl ether and petroleum ether. The solid was isolated by centrifugation and purified by reversed-phase prep-HPLC using C18 and an optimal gradient of buffer $\mathrm{A}\left(\mathrm{H}_{2} \mathrm{O} 95 \%\right.$, ACN 5\%, TFA 0.1\%) vs. buffer B (ACN 95\%, $\mathrm{H}_{2} \mathrm{O} 5 \%$, TFA $0.1 \%)$ to afford $6(51 \mathrm{mg}, 35 \%$, based on the purification of $\sim 100 \mathrm{mg}$ of the crude product 
by prep-HPLC). ${ }^{1} \mathrm{H}$ NMR (400 MHz, $\left.\mathrm{CDCl}_{3}\right): \delta 7.85(\mathrm{~d}, J=7.3 \mathrm{~Hz}$, aromatic $\mathrm{H}, 1 \mathrm{H}), 7.53-7.22$ $(\mathrm{m}$, aromatic H, 8H), $6.50(\mathrm{~d}, J=8.8 \mathrm{~Hz}, 1 \mathrm{H}), 5.72(\mathrm{dd}, J=8.9 \mathrm{~Hz}, J=4.7 \mathrm{~Hz}, \beta$-lactam C-H, $1 \mathrm{H}), 4.90\left(\mathrm{~d}, J=4.7 \mathrm{~Hz}, \beta\right.$-lactam C-H, 1H), 3.97-3.44 (m, aliphatic H, 8H), ${ }^{13} \mathrm{C}$ NMR (101 MHz, DMSO-d $\left.d_{6}\right): \delta 195.06,171.36,165.01,163.45,136.24,135.91,133.78,129.44,129.15$, $128.79,128.63,127.36,126.90,125.49,59.37,58.22,42.03,38.20,33.70,27.45$. HRMS (ESI): $[\mathrm{M}-\mathrm{H}]^{-}$calculated: 481.0897, found: 481.0863 .

General procedure for the synthesis of compounds 8-13. To a solution of $\mathrm{BF}_{3} . \mathrm{OEt}_{2}(2.6$ $\mathrm{mL}, 21.3 \mathrm{mmol}, 3.0$ eq. $)$ in acetonitrile $(10 \mathrm{~mL})$ were added the corresponding thiols $(10.7 \mathrm{mmol}$, 1.5 eq.) and 7-ACA (1.9 g, $7.1 \mathrm{mmol}, 1.0$ eq.) successively. The mixture was stirred at $45-50^{\circ}$ for $2 \mathrm{~h}$ after which it was diluted with water and $\mathrm{pH}$ was adjusted to 4 by adding $28 \%$ ammonium hydroxide solution. The precipitate was filtered off and washed with cold water and acetone respectively. The crude product $(1.0 \mathrm{~g})$ was added to a mixture of saturated bicarbonate solution $(6 \mathrm{~mL})$ and acetone $(9 \mathrm{~mL})$. Then phenylacetyl chloride (2.0 eq.) was added dropwise and the mixture was stirred overnight at room temperature. Diluting the mixture with water followed by acidification to $\mathrm{pH} 2.0$ using $1.0 \mathrm{M} \mathrm{HCl}$ resulted in a white solid which was filtered off and washed with minimum water and ether respectively. The crude material was purified by reversed-phase prep-HPLC using $\mathrm{C} 18$ and an optimal gradient of buffer $\mathrm{A}\left(\mathrm{H}_{2} \mathrm{O} 95 \%, \mathrm{ACN} 5 \%\right.$, TFA $0.1 \%$ ) vs. buffer $\mathrm{B}\left(\mathrm{ACN} 95 \%, \mathrm{H}_{2} \mathrm{O} 5 \%\right.$, TFA $\left.0.1 \%\right)$. The quantities and yields below are reported based on the purification of $100 \mathrm{mg}$ of the crude product by prep-HPLC.

Compound 8. $40 \mathrm{mg}$ (26\%, over two steps). ${ }^{1} \mathrm{H}$ NMR (400 MHz, DMSO- $\left.d_{6}\right)$ : diastereomeric mixture $\delta 9.07$ (apparent t, $1.8 \mathrm{H}), 7.44-7.21(\mathrm{~m}$, aromatic H, 9H), 5.61 (m, $\beta$ lactam C-H, 1.8H), 5.04 (d, $J=4.8 \mathrm{~Hz}, \beta$-lactam C-H, 0.8H), 4.88 (d, $J=4.7$ Hz, $\beta$-lactam C-H, 1H), 4.65 (apparent d, aliphatic $\mathrm{C}-\mathrm{H}, 1.8 \mathrm{H}), 3.69-3.32$ (m, aliphatic $\left.\mathrm{CH}_{2}, 10.8 \mathrm{H}\right),{ }^{13} \mathrm{C}$ NMR (101 MHz, DMSO-d $)_{6}$ : $\delta 172.05,171.98,171.45,171.43,164.99,163.52,163.48,137.83,137.54$, $136.29,129.51,129.05,128.94,128.89,128.76,128.71,128.31,127.24,126.97,125.65,125.60$, 59.38, 58.16, 52.63, 52.60, 42.06, 34.12, 33.76, 27.51, 27.44, HRMS (ESI): [M-H]- calculated: 497.0847, found: 497.0842 .

Compound 9. $69 \mathrm{mg}$ (47\%, over two steps). ${ }^{1} \mathrm{H}$ NMR (400 MHz, DMSO- $\left.d_{6}\right): \delta 9.14(\mathrm{~d}, J$ $=8.3 \mathrm{~Hz}, \mathrm{~N}-\mathrm{H}, 1 \mathrm{H}), 7.39-7.13(\mathrm{~m}$, aromatic H, 10H), $5.67(\mathrm{dd}, J=8.3,4.7 \mathrm{~Hz}, \beta$-lactam C-H, 1H), 5.08 (d, $J=4.8 \mathrm{~Hz}, \beta$-lactam C-H, 1H), 3.90-3.47 (m, aliphatic H, 7H), 3.04 (dd, $J=13.7$, $9.8 \mathrm{~Hz}$, aliphatic $\mathrm{H}, 1 \mathrm{H}), 2.93(\mathrm{dd}, J=13.7,5.8 \mathrm{~Hz}$, aliphatic $\mathrm{H}, 1 \mathrm{H}),{ }^{13} \mathrm{C} \mathrm{NMR}(101 \mathrm{MHz}$, 
DMSO- $\left.d_{6}\right): \delta 172.61,170.99,164.66,163.08,138.14,135.84,129.03,129.01,128.27,128.23$, $127.45,126.55,126.50,124.95,58.96,57.75,48.23,41.58,38.16,33.38,26.97$, HRMS (ESI): $[\mathrm{M}-\mathrm{H}]^{-}$calculated: 511.1003, found: 511.1000 .

Compound 10. $33 \mathrm{mg}$ (43\%, over two steps). ${ }^{1} \mathrm{H}$ NMR (400 MHz, DMSO- $\left.d_{6}\right): \delta 9.12$ (d, $J=8.4 \mathrm{~Hz}, \mathrm{~N}-\mathrm{H}, 1 \mathrm{H}), 7.37-7.17$ (m, aromatic H, 10H), 5.65 (dd, $J=8.4,4.7 \mathrm{~Hz}, \beta$-lactam C-H, 1H), 4.98 (d, $J=4.8 \mathrm{~Hz}, \beta$-lactam C-H, 1H), 3.73-3.33 (m, aliphatic H, 7H), 3.07 (dd, $J=13.8$, $8.6 \mathrm{~Hz}$, aliphatic $\mathrm{H}, 1 \mathrm{H}), 2.89(\mathrm{dd}, J=13.8,7.1 \mathrm{~Hz}$, aliphatic $\mathrm{H}, 1 \mathrm{H}),{ }^{13} \mathrm{C}$ NMR $(101 \mathrm{MHz}$, DMSO- $\left.d_{6}\right): \delta 173.36,171.43,165.03,163.48,138.76,136.29,129.61,129.50,128.71,128.67$, $126.98,126.62,125.77,59.38,58.19,47.85,42.07,37.81,33.57,27.10$, HRMS (ESI): $[\mathrm{M}+\mathrm{H}]^{+}$ calculated: 513.1154 , found: 513.1151 .

Compound 11. $82 \mathrm{mg}$ (74\%, over two steps). ${ }^{1} \mathrm{H}$ NMR (400 MHz, DMSO- $\left.d_{6}\right): \delta 9.13$ (d, $J=8.3 \mathrm{~Hz}, \mathrm{NH}, 1 \mathrm{H}), 7.35-7.22(\mathrm{~m}$, aromatic H, $5 \mathrm{H}), 5.65$ (dd, $J=8.3 \mathrm{~Hz}, J=4.7 \mathrm{~Hz}, \beta$-lactam C-H, 1H), 5.06 (d, $J=4.7 \mathrm{~Hz}, \beta$-lactam C-H, 1H), 3.79-3.47 (m, aliphatic H, 8H), ${ }^{13} \mathrm{C}$ NMR (101 MHz, DMSO- $\left.d_{6}\right): \delta 171.41,165.11,163.62,138.81,136.29,129.49,129.34,128.89,128.69$, $128.22,127.37,126.96,125.25,59.40,58.36,42.06,35.81,33.78,27.44$. HRMS (ESI): $[\mathrm{M}+\mathrm{H}]^{+}$ calculated 455.1099, found: 455.1098 .

Compound 12. $79 \mathrm{mg}$ (68\%, over two steps). ${ }^{1} \mathrm{H}$ NMR (400 MHz, DMSO- $\left.d_{6}\right): \delta 9.15$ (d, $J=8.3 \mathrm{~Hz}, \mathrm{~N}-\mathrm{H}, 1 \mathrm{H}), 7.33-7.19$ (m, aromatic H, 10H), 5.65 (dd, $J=8.4,4.7 \mathrm{~Hz}, \beta$-lactam C-H, $1 \mathrm{H}$ ), 5.13 (d, $J=4.7, \beta$-lactam C-H, 1H), 3.82-3.49 (m, aliphatic H, 6H), 2.85-2.65 (m, aliphatic $\mathrm{H}, 4 \mathrm{H}) .{ }^{13} \mathrm{C}$ NMR $\left(101 \mathrm{MHz}, \mathrm{DMSO}-d_{6}\right): \delta 171.45,165.20,163.73,140.89,136.30,129.50$, 129.29, 129.01, 128.78, 128.70, 126.97, 126.64, 125.11, 59.40, 58.51, 42.07, 36.30, 32.95, 32.44, 27.39, HRMS (ESI): $[\mathrm{M}+\mathrm{H}]^{+}$calculated: 469.1256 , found: 469.1256 .

Compound 13. $88 \mathrm{mg}$ (27\%, over two steps). ${ }^{1} \mathrm{H}$ NMR (400 MHz, DMSO- $\left.d_{6}\right): \delta 9.14$ (d, $J=8.3 \mathrm{~Hz}, \mathrm{~N}-\mathrm{H}, 1 \mathrm{H}), 7.34-7.21(\mathrm{~m}$, aromatic $\mathrm{H}, 5 \mathrm{H}), 5.65$ (dd, $J=8.3 \mathrm{~Hz}, J=4.7 \mathrm{~Hz}$, $\beta$-lactam C-H, 1H), 5.11 (d, $J=4.8 \mathrm{~Hz}, \beta$-lactam C-H, 1H), 3.73-3.20 (m, aliphatic H, 8H), ${ }^{13} \mathrm{C}$ NMR (101 MHz, DMSO- $d_{6}$ ): $\delta 170.55,170.44,164.10,162.47,135.31,128.51,127.72,126.48,125.99$, 124.48, 58.43, 57.31, 41.10, 32.94, 32. 74, 26.42. HRMS (ESI): $[\mathrm{M}+\mathrm{H}]^{+}$calculated: 423.0685, found: 423.0702 .

Compound 15. 7-ADCA $(\mathbf{1 4}, 2.14 \mathrm{~g}, 10 \mathrm{mmol})$ was dissolved in saturated bicarbonate solution $(20 \mathrm{~mL})$ to which phenylacetyl chloride $(1.5 \mathrm{~mL}, 11.3 \mathrm{mmol})$ dissolved in acetone $(10$ $\mathrm{mL}$ ) was added in several portions. The mixture was stirred overnight at room temperature, then 
acidified to $\mathrm{pH} 2.0$ using $1 \mathrm{M} \mathrm{HCl}$. The precipitate was filtered off and washed with minimum amount of cold water. The crude was purified by reversed-phase prep-HPLC using C18 and an optimal gradient of buffer A $\left(\mathrm{H}_{2} \mathrm{O} 95 \%\right.$, ACN 5\%, TFA 0.1\%) vs. buffer B (ACN 95\%, $\mathrm{H}_{2} \mathrm{O} 5 \%$, TFA $0.1 \%$ ). ( $85 \mathrm{mg}, 75 \%$, based on the purification of $\sim 100 \mathrm{mg}$ of the crude product by prepHPLC). ${ }^{1} \mathrm{H}$ NMR (400 MHz, DMSO- $\left.d_{6}\right): \delta 9.09(\mathrm{~d}, J=8.2 \mathrm{~Hz}, \mathrm{NH}, 1 \mathrm{H}), 7.33-7.21$ (m, aromatic H, 5H), 5.60 (dd, $J=8.2 \mathrm{~Hz}, J=4.6 \mathrm{~Hz}, \beta$-lactam C-H, $1 \mathrm{H}), 5.03$ (d, J = 4.7 Hz, $\beta$-lactam C-H, $1 \mathrm{H}), 3.61-3.35$ (m, aliphatic H, 4H), 2.03 (s, methyl, 3H), ${ }^{13} \mathrm{C}$ NMR (101 MHz, DMSO- $d_{6}$ ): $\delta$ 171.44, 164.82, 163.98, 136.33, 130.21, 129.48, 128.68, 126.93, 123.21, 59.33, 57.56, 42.03, 29.40, 19.87. HRMS (ESI): $[\mathrm{M}+\mathrm{H}]^{+}$calculated: 333.0909, found: 333.0917.

Enzyme production and purification. The procedures for the overexpression and purification of IMP-1, IMP-28, NDM-1, and VIM-2 have been described in detail in the a previous publication. ${ }^{12,18}$

Enzymatic preparation of $\boldsymbol{8 H}$ and $\mathbf{9 H}$. Compounds 8 and $\mathbf{9}(2 \mathrm{mM}$ each) was incubated with NDM-1 $(187 \mathrm{nM})$ at room temperature in $50 \mathrm{mM}$ HEPES-NaOH, pH 7.2 supplied with $1 \mu \mathrm{m}$ $\mathrm{ZnSO}_{4}$ and $0.01 \%$ triton $\mathrm{X}-100$. The progress of hydrolysis was monitored by LC-MS (Figure S7). After $2 \mathrm{~h}$ the conversion was complete, and compounds $\mathbf{8 H}$ and $\mathbf{9 H}$ were separated from the enzyme by spin-filtration ( $3 \mathrm{~K}$ filter cutoff, Amicon) at $12000 \mathrm{rpm}$ for $5 \mathrm{~min}$.

Enzyme inhibition assay. The cephalosporin derivatives were tested for their inhibitory activity against NDM-1, VIM-2 and IMP-28 using the chromogenic substrate nitrocefin. The assay buffer was $50 \mathrm{mM}$ HEPES pH 7.2, supplemented with $1 \mu \mathrm{M} \mathrm{ZnSO}_{4}$ and $0.01 \%$ triton X-100. Briefly, on a flat-bottom polystyrene 96-well microplate NDM-1 (6 nM), VIM-2 (8 nM) or IMP-28 $(1 \mathrm{nM})$ were incubated with various concentrations of the test compounds for $15 \mathrm{~min}$ at $25{ }^{\circ} \mathrm{C}$. Nitrocefin $\left(10 \mu \mathrm{M}, \sim 2 \times K_{\mathrm{M}}\right)$ was added to the wells and absorption at $492 \mathrm{~nm}$ was immediately monitored on a TECAN Spark microplate reader over 30 scan cycles. The initial velocity data were used for $\mathrm{IC}_{50}$ curve-fitting using GraphPad Prism 7. All the compounds were tested in 3 independent replicates.

Determination of the kinetic parameters of cephalosporin conjugates. Hydrolysis of the cephalosporin conjugates was monitored on a Tecan Spark microplate reader using 
UV-transparent 96-well plates (UV-Star ${ }^{\circledR}$, Greiner). Various concentrations of the test compounds were dissolved in $50 \mathrm{mM}$ HEPES-NaOH, pH 7.2 supplied with $1 \mu \mathrm{m} \mathrm{ZnSO}_{4}$ and $0.01 \%$ triton X-100. Followed by the addition of MBLs dissolved in the same buffer, absorption at $260 \mathrm{~nm}$ was measured immediately over $30-40$ scan cycles at $25{ }^{\circ} \mathrm{C}$. The obtained initial velocity data were plotted against substrate concentration, and $K_{\mathrm{M}}$ and $V_{\max }$ were determined using Michaelis-Menten fitting model on GraphPad Prism 7.

MIC determination and synergy assays. Minimum inhibitory concentration (MIC) of the test compounds were determined following the guidelines published by clinical and laboratory standards institute (CLSI) and as described earlier. ${ }^{11}$ Synergy between the cephalosporin derivatives and $\beta$-lactam antibiotics were evaluated by the following protocol: $\beta$-lactam antibiotics dissolved in Mueller-Hinton broth (MHB) with the concentration corresponding to $4 \times$ MIC was added to polypropylene 96 -well microplates and serially diluted $(25 \mu \mathrm{L} /$ well). Then each 3 columns received a fixed concentration of the test compounds dissolved in MHB (25 $\mu \mathrm{L} /$ well). Multiple concentrations of the test compounds were evaluated this way. Finally, bacterial suspensions grown to the $\mathrm{OD}_{600}$ of 0.5 were diluted 100x in MHB before adding to the plate $(50 \mu \mathrm{L} /$ well $)$. The microplates were then covered with breathable seals and incubated overnight with shaking at $37^{\circ} \mathrm{C}$ for $15-20 \mathrm{~h}$. Dipicolinic acid was used as positive control.

Stability analysis in MHB. The solutions of the test compounds (1 mM) in MHB were incubated at $37^{\circ} \mathrm{C}$ for $15 \mathrm{~h}$. Then, $100 \mu \mathrm{L}$ of the MHB solution was precipitated by adding to acetonitrile $(200 \mu \mathrm{L})$ supplied with $2 \mathrm{mM}$ benzocaine, vortexed and centrifuged (12000 rpm, $5 \mathrm{~min})$. The supernatant was analyzed by reversed-phase analytical HPLC using a $\mathrm{C}_{18}$ column and an optimal gradient of buffer A $\left(\mathrm{H}_{2} \mathrm{O} 95 \%\right.$, ACN 5\%, TFA 0.1\%) vs. buffer B (ACN 95\%, $\mathrm{H}_{2} \mathrm{O} 5 \%$, TFA $0.1 \%)$. The detector wavelength was set at $254 \mathrm{~nm}$.

Isothermal titration calorimetry. The ITC titrations were performed on a PEAQ-ITC calorimeter (Malvern). All the test compounds and zinc sulfate were dissolved in $20 \mathrm{mM}$ Tris- $\mathrm{HCl}$ buffer ( $\mathrm{pH}$ 7.0). The experiments consisted of titrating $2 \mathrm{mM}$ zinc sulfate through $19 \times 2.0 \mu \mathrm{L}$ aliquots (except the first aliquot which was $0.4 \mu \mathrm{L}$ ) into $200 \mu \mathrm{M}$ solutions of the cephalosporin conjugates incubated with NDM-1 (187 nM) for $2 \mathrm{~h}$ at room temperature. Experiments were performed at $25{ }^{\circ} \mathrm{C}$ with $150 \mathrm{~s}$ interval between titrations and reference power was set at 
$10.0 \mu \mathrm{cal} / \mathrm{s}$. Data was analyzed using Microcal PEAQ-ITC analysis software. In separate experiments, upon the titration of zinc sulfate into the solutions of cephalosporin conjugates or NDM-1, no binding interaction was observed.

NMR-based monitoring of the enzymatic hydrolysis. The cephalosporin conjugates dissolved DMSO- $d_{6}$ were diluted in deuterated PBS (pH 7.4) or deuterated 20 mM HEPES (pH 7.4) each supplemented with $1 \mu \mathrm{M} \mathrm{ZnSO}_{4}$. IMP-28 was added to the solution and the final concentration of the enzyme, test compounds and DMSO were $320 \mathrm{nM}, 1 \mathrm{mg} / \mathrm{mL}$ and $1 \%$ respectively. Following incubation at $25{ }^{\circ} \mathrm{C}$, the ${ }^{1} \mathrm{H}-\mathrm{NMR}$ spectra were measured on a Bruker $400 \mathrm{MHz}$ spectrometer in various time points.

LCMS-based monitoring of the enzymatic hydrolysis. The cephalosporin conjugates were dissolved in $20 \mathrm{mM}$ HEPES buffer ( $\mathrm{pH}$ 7.2) supplemented with $1 \mu \mathrm{M} \mathrm{ZnSO} \mathrm{Zn}_{4}$ and $0.01 \%$ triton $\mathrm{X}-100$. IMP-28 was added to the solution and the final concentration of the enzyme, test compounds and DMSO were $320 \mathrm{nM}, 1 \mathrm{mg} / \mathrm{mL}$ and $1 \%$ respectively. Following incubation at 25 ${ }^{\circ} \mathrm{C}$ and in different time points, the solution was diluted in ACN $(1: 2 \mathrm{v} / \mathrm{v})$ and centrifuged at $12000 \mathrm{rpm}$ for $5 \mathrm{~min}$. The supernatant was analyzed on an LCMS-8040 triple quadrupole liquid chromatograph mass spectrometer (LC-MS/MS, Shimadzu) using a C18 column ( $3 \mu \mathrm{m}, 3.0 \times 150$ $\mathrm{mm}$, Shimadzu) and a gradient of $5-100 \%$ pure acetonitrile against $0.5 \%$ formic acid.

In silico studies. All computational modelling was performed in the Schrodinger Suite version 2019-4. ${ }^{19}$ Figures were generated using PyMOL. 3D coordinates of the ligands were generated using LigPrep ${ }^{20}$ with the OPLS3e forcefield. ${ }^{21}$ Protein structures for IMP-1 (PDB ID: 1dd6) ${ }^{16}$ and NDM-1 (PDB ID: 4RL2) ${ }^{15}$ were prepared using the Protein Preparation Wizard. ${ }^{22,23}$ Thereafter, compounds were docked using GLIDE-SP. ${ }^{24}$ A maximum common substructure constraint for all product compounds was used, which was derived from cephalexin coordinates in PDB ID 4RL2. The substrate compounds were docked without using constraints. The best pose was maintained according to GLIDE docking score and visual inspection of the poses. 


\section{ASSOCIATED CONTENT}

\section{Supporting Information}

Analytical-spectral data including ${ }^{1} \mathrm{H}$ NMR, ${ }^{13} \mathrm{C}$ NMR and HPLC traces, $\mathrm{IC}_{50}$ curves, stability data, enzymatic degradation monitored by ${ }^{1} \mathrm{H}$ NMR and LCMS, and docking figures.

\section{Acknowledgements}

Funding was provided by the European Research Council (ERC consolidator grant to NIM, grant agreement no. 725523). 


\section{References}

(1) Spencer, J., Read, J., Sessions, R. B., Howell, S., Blackburn, G. M., and Gamblin, S. J. (2005) Antibiotic Recognition by Binuclear Metallo- $\beta$-Lactamases Revealed by X-ray Crystallography. J. Am. Chem. Soc. 127, 14439-14444.

(2) King, D. T., Worrall, L. J., Gruninger, R., and Strynadka, N. C. J. (2012) New Delhi Metallo$\beta$-Lactamase: Structural Insights into $\beta$-Lactam Recognition and Inhibition. J. Am. Chem. Soc. 134, 11362-11365.

(3) Drawz, S. M., and Bonomo, R. A. (2010) Three decades of beta-lactamase inhibitors. Clin. Microbiol. Rev. 23, 160-201.

(4) Bonomo, R. A., Burd, E. M., Conly, J., Limbago, B. M., Poirel, L., Segre, J. A., and Westblade, L. F. (2017) Carbapenemase-Producing Organisms: A Global Scourge. Clin. Infect. Dis. 66, 1290-1297.

(5) McGeary, R. P., Tan, D. T., and Schenk, G. (2017) Progress toward inhibitors of metallo- $\beta$ lactamases. Future Med. Chem. 9, 673-691.

(6) Groundwater, P. W., Xu, S., Lai, F., Váradi, L., Tan, J., Perry, J. D., and Hibbs, D. E. (2016) New Delhi metallo- $\beta$-lactamase-1: Structure, inhibitors and detection of producers. Future Med. Chem. 8, 993-1012.

(7) Tehrani, K. H. M. E., and Martin, N. I. (2018) $\beta$-lactam/ $\beta$-lactamase inhibitor combinations: an update. Medchemcomm 9, 1439-1456.

(8) Fast, W., and Sutton, L. D. (2013) Metallo- $\beta$-lactamase: Inhibitors and reporter substrates. Biochim. Biophys. Acta - Proteins Proteomics 1834, 1648-1659.

(9) Ju, L. C., Cheng, Z., Fast, W., Bonomo, R. A., and Crowder, M. W. (2018) The Continuing Challenge of Metallo- $\beta$-Lactamase Inhibition: Mechanism Matters. Trends Pharmacol. Sci. 39, $635-647$.

(10) Rotondo, C. M., and Wright, G. D. (2017) Inhibitors of metallo- $\beta$-lactamases. Curr. Opin. Microbiol. 39, 96-105.

(11) Tehrani, K. H. M. E., and Martin, N. I. (2017) Thiol-Containing Metallo- $\beta$-Lactamase Inhibitors Resensitize Resistant Gram-Negative Bacteria to Meropenem. ACS Infect. Dis. 3, 711717.

(12) van Haren, M. J., Tehrani, K. H. M. E., Kotsogianni, I., Wade, N., Brüchle, N., Mashayekhi, V., and Martin, N. I. (2020) Cephalosporin prodrug inhibitors overcome metallo b lactamase 
driven antibiotic resistance. Chem. - A Eur. J. DOI: 10.1002/chem.202004694.

(13) Strijtveen, B., and Kellogg, R. M. (1986) Synthesis of (racemization prone) optically active thiols by SN2 substitution using cesium thiocarboxylates. J. Org. Chem. 51, 3664.

(14) Hanessian, S., and Wang, J. (1993) Design and synthesis of a cephalosporin-carboplatinum prodrug activatable by a $\beta$-lactamase. Can. J. Chem. 71, 896-906.

(15) Feng, H., Ding, J., Zhu, D., Liu, X., Xu, X., Zhang, Y., Zang, S., Wang, D.-C., and Liu, W. (2014) Structural and Mechanistic Insights into NDM-1 Catalyzed Hydrolysis of Cephalosporins. J. Am. Chem. Soc. 136, 14694-14697.

(16) Concha, N. O., Janson, C. A., Rowling, P., Pearson, S., Cheever, C. A., Clarke, B. P., Lewis, C., Galleni, M., Frère, J.-M., Payne, D. J., Bateson, J. H., and Abdel-Meguid, S. S. (2000) Crystal Structure of the IMP-1 Metallo $\beta$-Lactamase from Pseudomonas aeruginosa and Its Complex with a Mercaptocarboxylate Inhibitor: Binding Determinants of a Potent, BroadSpectrum Inhibitor,. Biochemistry 39, 4288-4298.

(17) Keltjens, R., Vadivel, S. K., De Vroom, E., Klunder, A. J. H., and Zwanenburg, B. (2001) A new convenient synthesis of 3-carboxycephems starting from 7-aminocephalosporanic acid (7ACA). European J. Org. Chem. 2001, 2529-2534.

(18) Tehrani, K. H. M. E., Brüchle, N. C. ., Wade, N., Mashayekhi, V., Pesce, D., van Haren, M., and I. Martin, N. (2020) Small molecule carboxylates inhibit metallo- $\beta$-lactamases and resensitize carbapenem-resistant bacteria to meropenem. ACS Infect. Dis. 6, 1366-1371.

(19) Schrödinger Release 2019-4: Maestro, Schrödinger, LLC, New York, NY, 2019.

(20) Schrödinger Release 2019-4: LigPrep, Schrödinger, LLC, New York, NY, 2019.

(21) Roos, K., Wu, C., Damm, W., Reboul, M., Stevenson, J. M., Lu, C., Dahlgren, M. K., Mondal, S., Chen, W., Wang, L., Abel, R., Friesner, R. A., and Harder, E. D. (2019) OPLS3e: Extending Force Field Coverage for Drug-Like Small Molecules. J. Chem. Theory Comput. 15, $1863-1874$.

(22) Madhavi Sastry, G., Adzhigirey, M., Day, T., Annabhimoju, R., and Sherman, W. (2013) Protein and ligand preparation: parameters, protocols, and influence on virtual screening enrichments. J. Comput. Aided. Mol. Des. 27, 221-234.

(23) Schrödinger Release 2020-4: Protein Preparation Wizard; Epik, Schrödinger, LLC, New York, NY, 2016; Impact, Schrödinger, LLC, New York, NY, 2016; Prime, Schrödinger, LLC, New York, NY, 2020. 
(24) Friesner, R. A., Banks, J. L., Murphy, R. B., Halgren, T. A., Klicic, J. J., Mainz, D. T., Repasky, M. P., Knoll, E. H., Shelley, M., Perry, J. K., Shaw, D. E., Francis, P., and Shenkin, P. S. (2004) Glide: A New Approach for Rapid, Accurate Docking and Scoring. 1. Method and Assessment of Docking Accuracy. J. Med. Chem. 47, 1739-1749. 